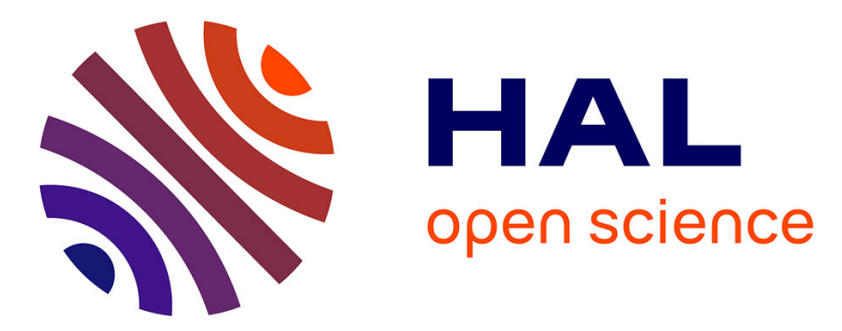

\title{
Experiments on radiative cooling of a shock-heated gas
}

S.P. Vaguin, Yu. A. Yacobi, V.V. Yakovlev, R.I. Soloukhin

\section{To cite this version:}

S.P. Vaguin, Yu. A. Yacobi, V.V. Yakovlev, R.I. Soloukhin. Experiments on radiative cooling of a shock-heated gas. Revue de Physique Appliquée, 1978, 13 (8), pp.399-403. 10.1051/rphysap:01978001308039900 . jpa-00244467

\section{HAL Id: jpa-00244467 https://hal.science/jpa-00244467}

Submitted on 1 Jan 1978

HAL is a multi-disciplinary open access archive for the deposit and dissemination of scientific research documents, whether they are published or not. The documents may come from teaching and research institutions in France or abroad, or from public or private research centers.
L'archive ouverte pluridisciplinaire HAL, est destinée au dépôt et à la diffusion de documents scientifiques de niveau recherche, publiés ou non, émanant des établissements d'enseignement et de recherche français ou étrangers, des laboratoires publics ou privés. 


\title{
EXPERIMENTS ON RADIATIVE COOLING OF A SHOCK-HEATED GAS
}

\author{
S. P. VAGUIN, Yu. A. YACOBI, V. V. YAKOVLEV \\ Institute for Pure and Applied Mechanics, USSR Academy of Sciences, Novosibirsk, USSR \\ and \\ R. I. SOLOUKHIN \\ Heat and Mass Transfer Institute, BSSR Academy of Sciences, Minsk, USSR
}

(Reçu le 27 février 1978, révisé le 24 avril 1978, accepté le 8 mai 1978)

\begin{abstract}
Résumé. - On passe en revue les résultats des études du refroidissement d'un plasma d'argon par pertes radiatives dans un tube à choc. Le gradient de densité électronique, $\mathrm{d} n_{\mathrm{e}} / \mathrm{d} x$, est utilisé comme paramètre pour déterminer les pertes radiatives du gaz réchauffé par une onde de choc. La distribution spatiale de la densité électronique est déterminée par une méthode interférométrique en utilisant une source de lumière infrarouge $(10,6 \mu)$. Ces études montrent qu'à l'extrémité de la zone où l'ionisation est faible, les valeurs mesurées de densité électronique et de température de gaz coïncident avec les données calculées à l'équilibre. Cela signifie que le rayonnement dans cette zone est faible et qu'en général, le refroidissement par pertes radiatives se situe dans la région d'écoulement à l'équilibre. La mesure des pertes radiatives montre que ces pertes dépassent considérablement les valeurs calculées pour le continuum transparent dans tout le domaine spectral et que l'émission sur des raies discrètes est la cause principale des pertes.
\end{abstract}

\begin{abstract}
The paper describes shock-tube data on radiative cooling of an argon plasma, the electron density gradient, $\mathrm{d} n_{\mathrm{e}} / \mathrm{d} x$, being a good indicator of the radiation lost by a shock-heated gas. The electron density profiles were obtained interferometrically with the use of an infrared light source $(10.6 \mu)$. The experimental results have shown that close to the end of the ionization relaxation zone, the measured electron densities and temperatures agree fairly well with the equilibrium calculations. This is an indication that the radiation yield has a weak effect in the relaxation zone and that the radiative losses in the equilibrium flow region should be taken into account. The measured losses are shown to be far in excess of those calculated for the continuum transparent throughout the whole spectral range, which is indicative of the discrete line radiation to be mainly responsible for the radiative losses.
\end{abstract}

Introduction. - Numerous systematic studies and detailed calculations of radiation energy transfer in a heated gas have been stimulated by various practical high-temperature gasdynamic problems. The radiation effects of the gasdynamic parameter profiles are represented in the energy conservation equation by the radiant-flux divergence [17] that depends on the radiation intensity and the spectral absorption coefficient of a medium. The radiation characteristics are, in their turn, defined by gasdynamic parameter distributions. On physical grounds, the radiant flux divergence is the difference between the radiant energy emitted per unit volume per unit time and the energy absorbed by this volume. The integro-differential equation for radiation transfer should be used to determine the radiation intensity field, and some simplifying assumptions are needed to solve the complex system of the transfer equations. Therefore, experimental verification of the solutions obtained will be required before they can be applied with confidence. The availability of shock-tube facilities has allowed accumulation of vast experimental data on spectral radiation characteristics of different gases [15] on radiative cooling of a shock-heated gas and on the relative contributions of continuum and spectral lines to radiation $[10,11,4,14]$. The shock tube studies show the essential effect of radiative cooling on the equilibrium shock-layer flow field parameters. This effect may be employed for experimental determination of the magnitude of integral radiation losses 
in terms of a time history of some shock-flow parameters. This approach is of great interest because of the complexity of direct measurements of the radiant flux over the whole spectrum.

This paper describes the experimental techniques and results of determining integral radiation losses in a shock-heated gas based on the recording of electron density profiles behind normal shock waves. The electron density is measured here because it is one of the most sensitive parameters varying with a gas state at high temperature. Experimental electron density profiles are attained by the IR laser-interferometry method (10.6 $\mu \mathrm{m}$ wavelength), which has improved the measurement sensitivity and, consequently, the integral radiation losses were found in the parameter region lacking experimental studies.

Principle. - After ionization relaxation ceases in a shocked noble gas, the gas state achieves its local thermodynamic equilibrium. Then, the electron density starts decreasing slowly, which occurs mainly due to radiation losses of the shock-heated gas volume. If radiation cooling is assumed to be the principal source of energy losses and the boundary layer effects are neglected, then the one-dimensional conservation equations for steady flow in the coordinate system associated with the shock wave front should be written as :

$$
\begin{gathered}
\mathrm{d}(\rho u) / \mathrm{d} x=0 \\
\rho u \mathrm{~d} u / \mathrm{d} x+\mathrm{d} p / \mathrm{d} x=0 \\
\rho u \mathrm{~d}\left(h+u^{2} / 2\right) / \mathrm{d} x+Q=0
\end{gathered}
$$

where $\rho, u, p, T$ are the density, velocity, pressure and temperature of a gas, respectively, and $x$ the distance along the shock tube axis recorded from the shock wave front. Here the gas flow parameters are treated as one-dimensional whereas $Q$, the loss of energy due to radiative transfer per unit volume of a gas per unit time, is generally three-dimensional. The radiative loss term is expressed as

$$
Q=\operatorname{div} \mathbf{S}=\int_{0}^{\infty} \mathrm{d} v \int_{\Omega} K_{v}\left(B_{v}-I_{v}\right) \mathrm{d} \Omega
$$

where $\mathbf{S}$ is the radiative energy flux. Integration is performed both over the whole frequency interval $(0<v<\infty)$ and space angle, $\Omega$, with regard to a real distribution of spectral radiation intensity, $I_{v}$, and the effective spectral absorption coefficient, $K_{v}$, corrected to include induced emission, throughout the whole light emitting gas volume. The Planck function, $B_{v}$, is defined in terms of the radiation frequency and temperature of a medium. Thus, light reabsorption leads, in a general case, to the non-uniformity of a gasdynamic parameter distribution. Consequently, the system of the conservation equations becomes three-dimensional. However, if in the total radiation loss balance the reabsorption is not predominant, then the one-dimensional system (1)-(3) may be used.

Under the conditions chosen, the specific enthalpy of a partially ionized gas, $h$, is determined by translational and ionization energy (excited atom energy may be ignored as it is evaluated to be less than 1 per cent of the ionization energy, the latter being of the order of 10-20 per cent of the translational energy)

$$
h=2.5(1+\alpha) k T / m_{\mathrm{a}}+\alpha I / m_{\mathrm{a}},
$$

where $T$ is the gas temperature, $I$ the atom ionization energy, $\alpha=n_{\mathrm{e}} /\left(n_{\mathrm{e}}+n_{\mathrm{a}}\right)$ the ionization degree, $n_{\mathrm{e} . \mathrm{a}}$ the electron and atom number density, respectively, $k$ the Boltzmann constant, $m_{\mathrm{a}}$ the atom mass. The system of equations (1)-(3) is completed by the gas state equation

$$
p=\left(n_{\mathrm{a}}+2 n_{\mathrm{e}}\right) k T
$$

and the ionization equilibrium (Saha) equation for a single-ionized plasma :

$$
n_{\mathrm{e}}^{2} / n_{\mathrm{a}}=2 Z_{\mathrm{i}}\left(\frac{2 \pi m_{\mathrm{e}} k T}{h^{2}}\right)^{3 / 2} \exp \left(\frac{I-\Delta I}{k T}\right),
$$

where $Z_{\mathrm{i}}$ is the ion partition function. In argon,

$$
Z_{\mathrm{i}} \simeq 4+2 \exp \left(-\frac{2060}{T}\right)
$$

and $\Delta I$ the ionization potential reduction due to the Coulomb particle interaction [3], $h$ the Planck constant, $m_{\mathrm{e}}$ the electron mass.

If the gas ionization degree is small $(\alpha \ll 1)$, the specific kinetic flow energy is also small compared with the specific enthalpy $\left(u^{2} / 2 \ll h\right)$ and if we assume that the gas flow temperature varies slightly in the equilibrium region (this is taken into account for differentiating the Saha equation), then from equation (3) in terms of (1)-(2), (5)-(7) the following expression may be derived for the radiative loss term :

$Q=\left|\left(\frac{5}{2} k T+I\right) \frac{p_{1}}{\left(n_{\mathrm{a}}+n_{\mathrm{e}}\right) k T_{1}}+\frac{5(k T)^{2} p_{1}}{m_{\mathrm{e}} n_{\mathrm{e}} I k T_{1}}\right| \frac{\mathrm{d} n_{\mathrm{e}}}{\mathrm{d} t}$,

where $\mathrm{d} t$ is the time interval recorder in the laboratory coordinate system, $\mathrm{d} t=\mathrm{d} x / D, D$ the shock wave velocity. Expression (8) is given in the form convenient for experimental determination of integral radiation losses. For this purpose on some plane behind a shock wave front, the thermodynamic parameters (temperature, electron and atom density) and electron density rates, $\mathrm{d} n_{\mathrm{e}} / \mathrm{d} t$, need to be known.

Experimental. - Experiments were made in a double-diaphragm shock tube $7.6 \mathrm{~cm}$ i.d. and the driven section was $3.4 \mathrm{~m}$ long. Helium gas electrically heated up to $250^{\circ} \mathrm{C}$ at a reservoir pressure of $150 \mathrm{~atm}$ was used in the driver for shock heating of a buffer 
gas, argon, in the intermediate section at a pressure of 0.05-0.2 atm. The 99.999 purity argon served as a test gas. The vacuum system ensured the pumping of the driven section to a bare pressure of $10^{-5}-10^{-6}$ torr. The experimental set up is shown in figure 1. Two ionization probes located near the test shock tube section and the electronic frequency meter were used for shock timing. The accuracy of the shock speed measurement was estimated to be better than $1 \%$.

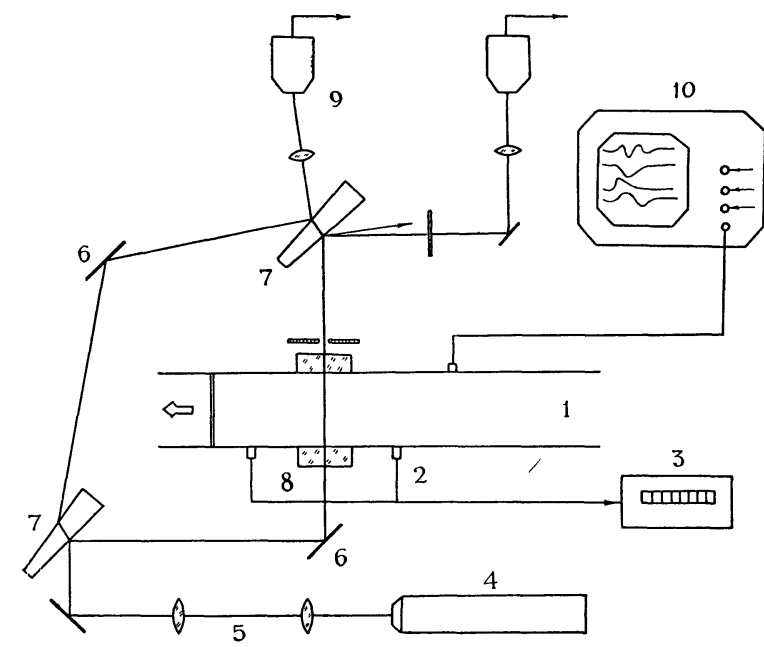

FIG. 1. - Schematic diagram of the experimental system : 1. shock tube ; 2 . ionization probes; 3 . time interval meter; $4 . \mathrm{CO}_{2}$ laser : 5. telescopic system; 6. Mach-Zehnder interferometer; 7. IR beam splitter (germanium); 8. barium fluorine window; 9. IRradiation $\mathrm{Ge}-\mathrm{Au}$ detector; 10 . oscilloscope.

The electron number density behind a shock front was measured with a Mach-Zehnder interferometer using photoelectric fringe shift recording. The $\mathrm{CO}_{2}$ laser operated at $10.6 \mu \mathrm{m}$ wavelength served as a light source. It is known that the use of an IR-source improves the sensitivity of the optical methods to the electron density. This has stimulated the development of various infrared diagnostic techniques in ionized gas studies $[8,9,12]$. A typical oscilloscope record of the interference fringe shift recorded by the liquid nitrogen cooled infrared $\mathrm{Ge}-\mathrm{Au}$ detector and the typical electron density profile calculated for this oscilloscope record are shown in figure 2 . The measuring error of maximum values of $n_{\mathrm{e}}$ was $1 \div 2 \%$. As shown in figure 1, the intensity of a beam passing through a shock-heated gas can also be detected thus allowing measurement of the test gas absorption coefficient at the $\mathrm{CO}_{2}$ laser frequency. Measurements of the absorption coefficient in an argon plasma sample enable one to determine the plasma temperature provided that the electron concentration is measured independently. On the other hand, the argon plasma temperature can be calculated based purely on the Saha equation if the electron concentration is known. Figure 3 shows a plot of the gas temperature distribution behind a shock wave as obtained by both methods, plasma absorption measu-
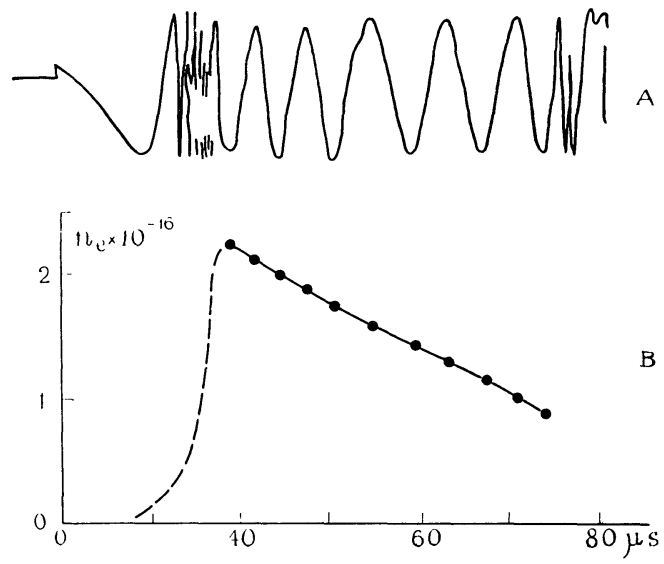

Fig. 2. - Interference oscilloscope record $(a)$ and calculated electron density profile $\left(n_{\mathrm{e}}\right)$ behind a shock wave $(b)(M=12.13$. $p_{1}=3$ torr).

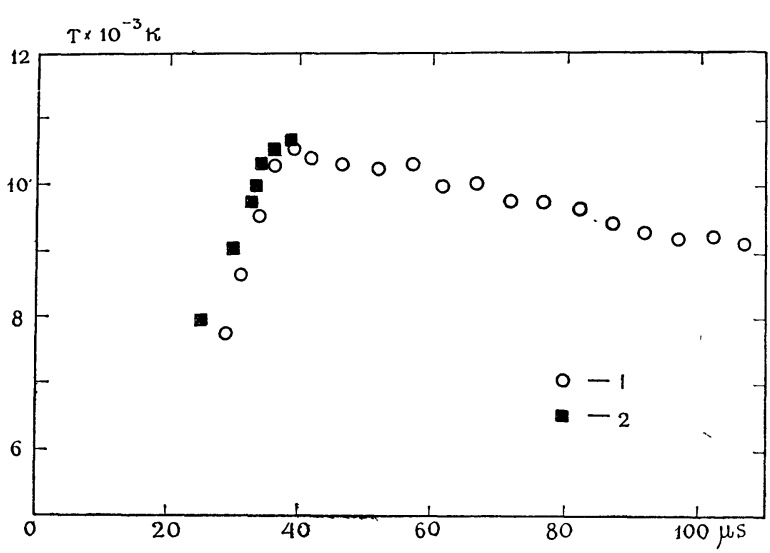

FIG. 3. - Argon plasma temperature profiles behind a shock wave, $M=12.19 ; p_{1}=3$ torr, as determined from the IR absorption (1) and electron density (2) measurements.

rement data and electron concentration measurements based on the Saha equation. For this 3 torr shot, with a shock Mach number of 12.19 , the equilibrium calculated temperature is $10630 \mathrm{~K}$. It is seen from figures 2 and 3 that behind the shock front in the ionization relaxation region, the electron density and gas temperature attain their maximum values, then drop slowly thereby undergoing, for $n_{\mathrm{e}}, 2-2.5$ time decrease before interacting with the contact surface.

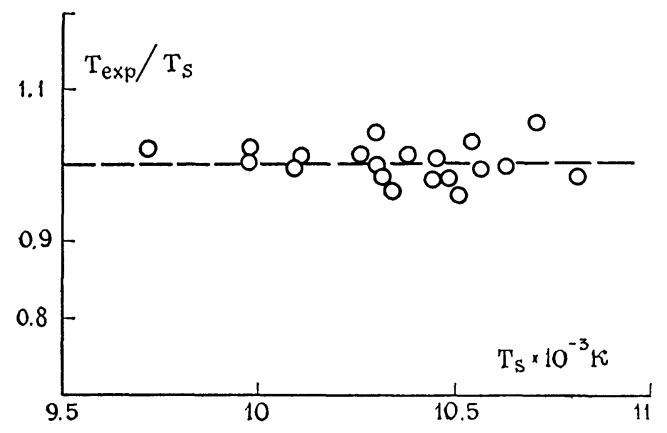

FIG. 4. - Measured maximum argon plasma temperatures behind a shock wave as a function of the equilibrium shocked gas temperature. 
Maximum values of $n_{\mathrm{e}}$ and plasma temperature were shown to coincide with the predicted equilibrium ones [13]. Figure 4 shows that the maximum temperature measurements agree fairly well with the equilibrium calculations. It follows that the experimental parameters attain their equilibrium values when ionization relaxation has ceased, thus pointing to small energy losses in this flow region.

Results and discussion. - The measured profiles of an electron density decrease behind the shock wave front were used for quantitative determination of integral radiation losses by formula (8). For this purpose the derivative, $\mathrm{d} n_{\mathrm{e}} / \mathrm{d} t$, was calculated near a maximum value of $n_{\mathrm{e}}$. The values of other parameters were taken as those calculated from the shock adiabatic. Since their change near maximum $n_{\mathrm{e}}$ appears to be small the uncertainty in $Q$ may be estimated to be no more than $5-10 \%$. The uncertainty associated with determining $\mathrm{d} n_{\mathrm{e}} / \mathrm{d} t$ by the experimental curve $n_{\mathrm{e}}(t)$ should also be taken into account. The total uncertainty of integral losses, $Q$, is $20-25 \%$.

Figure 5 gives the experimental values of integral radiation losses in argon at initial pressures of 3 and 5 torr as a function of the shock Mach number. Despite a small Mach number range and the above uncertainty, it is clearly seen that $Q$ increases with $M$, i.e. with gas temperature and pressure.

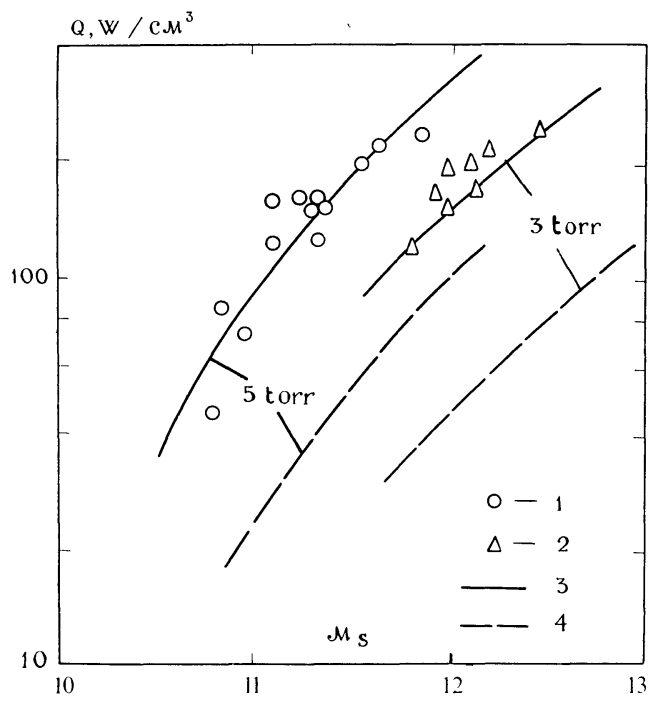

FIG. 5. - Measurements and calculations of radiation losses, $Q$, of shock-heated argon behind a shock front at different initial pressures, $p_{1}$, as a function of the shock Mach number. 1. experiment, $p_{1}=5$ torr; 2 . experiment, $p_{1}=3$ torr; 3 . calculations, equation $(9) ; 4$. calculations, only the contribution of the continumm is included.

In noble gases the radiation losses are attributed to spectral lines (bound-bound electron transitions) and continuum (free-bound and free-free electron transitions in the fields of ions and atoms) [17]. The noble gas radiation in the continuum can he easily found [2] since the summation over all levels is replaced hy integration when calculating the photorecombination radiation. For argon, only electron transitions to the ground and $4 \mathrm{~s}$ states may be taken into account separately. For experimental comparison, the value of the integral radiation losses in the continuum (only) is estimated under the assumption that, except photorecombination radiation to the ground state, radiation is transparent. The results are shown in figure 5 by the dashed lines and lie considerably below the experimental data. This confirms the results of Yakubov [16], from which it follows that the spectral line contribution to radiation is the main source of radiation losses in argon (the shocked gas state, $\left.p=0.8-1 \mathrm{~atm}, T=10^{4} \mathrm{~K}\right)$. Including all energy levels hampers exact calculation of the integral radiation losses and requires a great deal of computer time.

According to our estimates, the plasma may be considered to be transparent for integral radiation. In this case the radiation losses are determined simply by the integral gas emissivity, $\varepsilon$, and are equal to $Q=4 \pi \varepsilon$. It is of interest to represent $\varepsilon$ in a simple analytical form. The contribution of the continuum is taken into account [2] and that of the spectral lines, by their spreading over the spectrum in accordance with the spectroscopic stability principle. In the work by Kozlov et al. [5] a simple formula is obtained for the total integral emissivity :

$\varepsilon=1.14 \times 10^{-26} \cdot n_{\mathrm{e}}^{2}\left(T / 10^{4}\right)^{1 / 2} \exp \left(\frac{h v_{\mathrm{th}}-\Delta I}{k T}\right)$

where $v_{\text {th }}$ is the limit frequency equal to a maximum threshold frequency of the photoeffect for the levels which are taken into account integrally. For argon $h v_{\text {th }}=2.85 \mathrm{eV}$. The formula (9) was shown to be valid within the temperature range between 8000 and $15000 \mathrm{~K}$ for a single-ionized plasma at $p=1 \mathrm{~atm}$. Figure 5 shows the comparison between the experimental and predicted data (solid lines). Their good coincidence gives the possibility of applying the simple method for calculating the integral emissivity even in that region of parameters where the spectral line radiation is predominant. The analysis of relation (9) shows that for small ionization, $\varepsilon$ is proportional to the gas pressure, similarly to the case when nonabsorbed line radiation is taken into account. This dependence allows reduction of the data obtained at various pressures to one pressure of interest, thus giving an opportunity to compare the data obtained at different pressures behind a shock wave with those found by other methods. As an example, figure 6 gives a plot of the integral losses versus temperature reduced to atmospheric pressure.

It is known that the gasdynamic parameter, variations behind a shock front are also associated with boundary layer effects. Shock tube flow is shown to be similar to that in a diverging nozzle of variable crosssection, $A(x)$ being dependent on the boundary layer 


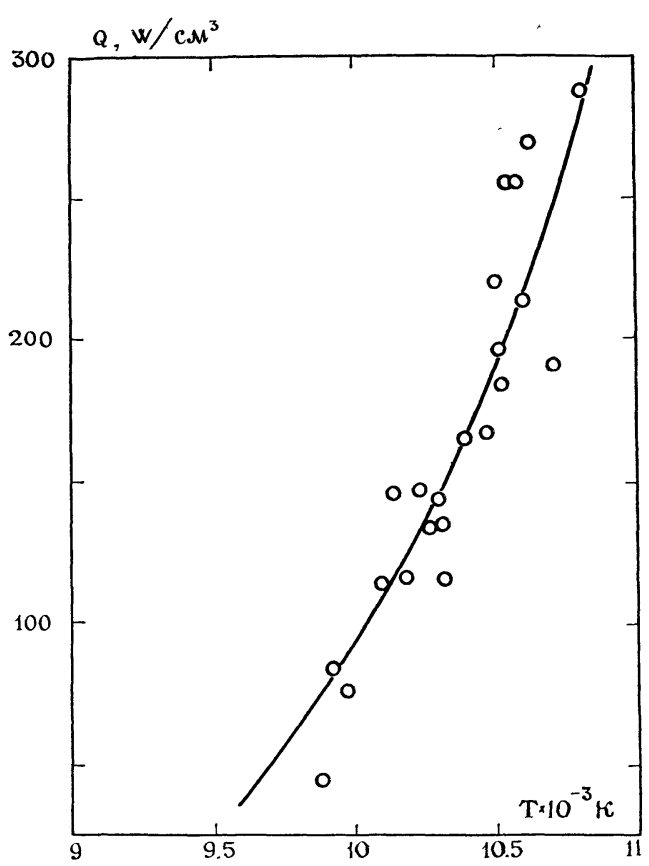

FIG. 6. - Radiation losses in the argon plasma as a function of temperature reduced to $1 \mathrm{~atm}$ pressure. 1. present experiments; 2. calculation by formula (9).

development, [7]. If the maximum distance between the shock wave front and contact surface in the steadystate flow regime is designated through $l_{\mathrm{m}}$, then the effective nozzle cross-section is described by a simple relation :

$$
A_{0} / A(x)=1-\left(x / l_{\mathrm{m}}\right)^{s}
$$

where $A_{0}$ is the shock tube channel cross-section. $s=0.5$ for a laminar boundary layer and $s=0.8$ for a turbulent one.

For typical experimental conditions, the viscosity effect on the gas flow in the shock tube was estimated. The system of equations (2), (3), (5)-(7) together with the mass conservation equation, $\rho u A=$ const. were solved numerically. The equilibrium parameters deter-

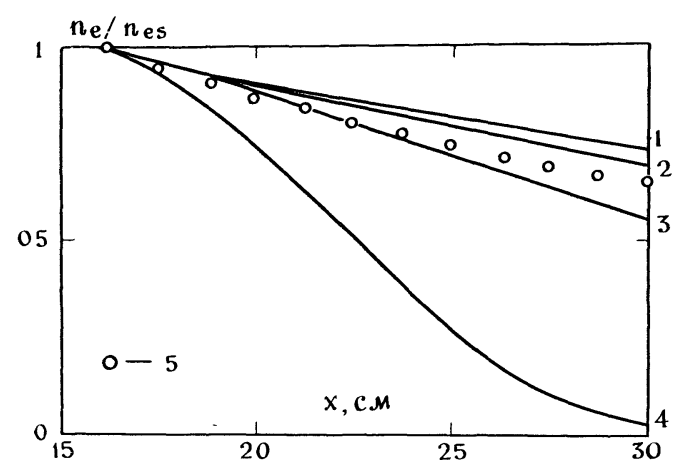

Fig. 7. - Comparison between the measured and calculated electron density profiles. 1 . without regard to the boundary layer development in a shock-tube ; $2,3,4$. with regard to the boundary layer effects (parameter $l_{\mathrm{m}}$ is equal to 200,100 and $50 \mathrm{~cm}$, respectively); 5. experimental data.

mined by the conservation equations for one-dimensional shock-tube flow served as the initial conditions. Since a finite length ionization relaxation zone exists behind the shock-front, the initial value of $x$ was taken equal to the experimental relaxation zone length. For argon, the parameter, $l_{\mathrm{m}}$, was extrapolated from the work of Mirels [6] to be about $200 \mathrm{~cm}$ for the range of conditions in this experiment. The integral radiation losses, $Q=4 \pi \varepsilon$, were calculated by formula (9). Figure 7 shows a comparison between the measured electron density profile in the equilibrium region behind a shock wave ( $p_{1}=3$ torr, $M=12.12$ ) and the predicted one at different $l_{\mathrm{m}}$ for the case of a laminar boundary layer. It is seen from figure 7 that at $l_{\mathrm{m}}=200 \mathrm{~cm}$ the boundary layer effects on the shock tube flow are insignificant. The same conclusion is valid for a turbulent boundary layer. This certainly confirms the applicability of the method for determining integral radiation losses in a shock-heated gas based on the one-dimensional flow approximation, the electron density decay determination providing a means of -sensitively detecting the integral radiation losses behind an ionizing shock wave.

\section{References}

[1] Biberman, L. M., Mnatsakanyan, A. Kh. and Yakubov, I. T., Usp. Fiz. Nauk 102 (1970) 431.

[2] Biberman, L. M. and Norman, G. E., Usp. Fiz. Nauk 91 (1967) 193.

[3] Griem, H. R., Phys. Rev. 128 (1962) 997.

[4] Horn, K. P., Wong, H. and Bershader, D., J. Plasma Physics 1 (1967) 157.

[5] Kozlov, G. I., Kuznetsov, V. A. and Masyukov, V. A., $Z h$. Eksp. Teor. Fiz. 66 (1974) 954.

[6] Mirels, H., Phys. Fluids 6 (1963) 1202.

[7] Mirels, H., Phys. Fluids 9 (1966) 1907.

[8] Offenberger, A. A. and Kerr, R. D., J. Appl. Phys. 43 (1972) 354.

[9] Offenberger, A. A., Kerr, R. D. and Smy, P. R., J. Appl. Phys. 43 (1972) 574.

[10] Petschek, H. E., Rose, P. H., Glick, H. S., Kane, A. and Kantrowitz, A., J. Appl. Phys. 26 (1955) 83.
[11] Sfvastyanflko. V. G. and Y ini bo). I T. Opt. Spektrosk. $16(1964) 3$.

[12] Soloukhin, R. I., Yacobi, Yu. A. and Yacovlev, V. I., Arch. Mech. 26 (1974) 637.

[13] Soloukhin, R. I., Yacobi, Yu. A. and Yacovlev, V. I., Fiz Gorenija Vzryva 13 (1977) 481.

[14] Stickford, Jr. G. H., AIAA J. 14 (1976) 421.

[15] Wilkerson, T. D., Koopman, D. M., Miller, M., Bengtson, R. and Charatis, G., Phys. Fluids Suppl. 12 (1969) 22.

[16] Yakubov, I. T., Opt. Spektrosk 19 (1965) 497.

[17] Zel'dovich, Ya. B. and Raizer, Yu. P., Physics of Shock Waves and High-Temperature Hydrodynamic Phenomena (2nd Edn. Izdatel'stvo Nauka, Moscow, 1966. Trans. 1967 ; Editors : W. D. Hayes and R. F. Probstein, Academic Press). 